\title{
Block copolymer electrolytes for fuel cells and secondary batteries, the small angle neutron scattering inputs
}

\author{
Laurent Rubatat
}

CNRS/ UNIV PAU \& PAYS ADOUR/ E2S UPPA, Institut des Sciences Analytiques et de PhysicoChimie pour l'Environnement et les Matériaux, IPREM UMR5254, 64000, Pau, France

\begin{abstract}
This paper aims at giving an overview on the importance of scattering, and more specifically neutron scattering, for probing the nanomorphology of polymer electrolytes made of block copolymers. Two types of self-assembled polymer electrolyte materials will be discussed: (i) the ionomer membranes used in fuel cell and (ii) the solid polyelectrolytes used in secondary batteries. Both are used to physically separate the electrodes in the respective electrochemical devices and are expected to have a high ion transport capacity so as good chemical and mechanical stabilities. Unfortunately, in most cases improving one property leads to the degradation of the others. Nonetheless, through block copolymers selfassembly it is possible to tackle this issue; indeed, antagonist properties can be decoupled and associated within controlled nano-morphologies. This aspect will be discussed and supported by examples based on published studies; in parallel useful scattering analytical tools and models will be presented along the paper and detailed in annex.
\end{abstract}

\section{Introduction}

Nowadays ion containing polymers materials are of major interest in devices such as fuel cells and secondary batteries. Ionomer membranes such as Nafion have proved their high potential to transport efficiently protons in low temperature fuel cells; and polymer electrolytes, such as polyethylene oxide (PEO), have demonstrated their capacity to well dissociate lithium salts and transport ions between batteries electrodes. The relatively low cost and ease to process, make polymer materials of great interest for such applications. Nevertheless, improvements are still necessary; for instance, the stability of ionomer membranes needs to be improved when cycled - swelling/drying cycles - in order to extend the fuel cell lifetime; also, the mechanical modulus of polymer electrolyte should be increased toward solid polyelectrolytes for a safer use of the batteries. Unfortunately, in most cases improving one property leads to the degradation of the others. One rout to decouple antagonist properties is through block copolymers self-assembly, where specific polymers with their one desirable property (e.g. ion conductivity, mechanical strength, etc.)

* Corresponding author: laurent.rubatat@univ-pau.fr 
are associated along a single polymer chain and later phase segregated and self-assembled into nano-domains to form the electrolyte material.

Block copolymer (BCP) self-assembly mechanisms are well observed experimentally[1] and well described theoretically [1-4] ( $c f$. figure 1). The nano-morphology is controlled essentially through the Flory-Huggins parameter, $\chi$, the total number of repeating units along the macromolecule, $N$, and the volume ratio of one of the blocks, $f$. Also, the macromolecular architecture (e.g. linear, star-like, comb-like, blocks number, blocks dispersity, etc.) has an impact on the self-assembly.[5,6] The variation of those parameters leads to a variety of structures such as lamellar, hexagonal packing of cylinders, spheres, and bicontinuous morphologies, for the most common as depicted on figure 1.[1] In the case of a charge free block copolymers, a single Flory-Huggins parameter, $\chi$, describes the properties of the system in both the ordered and disordered phases, corresponding respectively to the strong $(\chi \cdot N>>10)$ and the weak $(\chi \cdot N<10)$ segregation limit, which are unified through the self-consistent mean field theory.[4] Considering a diblock copolymer $\mathrm{AB}$ constituted of a block $\mathrm{A}$ and a block $\mathrm{B}, \chi_{A B}$ depends on temperature and on the chemical nature of both blocks and quantify their degree of incompatibility. Then $\chi_{A B}$ can be expressed as follow: [1]

$$
\chi_{A B}=\left(\frac{z}{k_{B} T}\right)\left[\epsilon_{A B}-\frac{1}{2}\left(\epsilon_{A A}+\epsilon_{B B}\right)\right]
$$

where $z$ is the number of nearest neighbors per repeat units in the polymer, $k_{B}$ is the Boltzman constant, $T$ the temperature, and $\varepsilon_{A B}$ is the interaction energy per repeat unit of A$\mathrm{B}, \varepsilon_{A A}$ for A-A and $\varepsilon_{B B}$ for B-B. A negative $\chi_{A B}$ indicates a free-energy drive towards mixing of $\mathrm{A}$ and $\mathrm{B}$, on the other hand a positive one indicates a clear repulsion between $\mathrm{A}$ and $\mathrm{B}$. Experimentally, rough estimations of $\chi$ can be obtained through contact angle measurements [7] or through solubility parameters.[8] Scattering techniques, such as small angle X-ray scattering (SAXS) and small angle neutron scattering (SANS) can also be exploited to extract the parameter; whether by the domain spacing in the ordered phase,[7] or by the behaviour of the structure factor in the disordered phase.[2, 9] This particular point will be discussed in the solid electrolytes section and developed in annex. Neutron reflectometry can be used to characterize the interface between both layers of homopolymers $\mathrm{A}$ and $\mathrm{B}$ which also depends on the $\chi_{A B}$ parameter.[7]

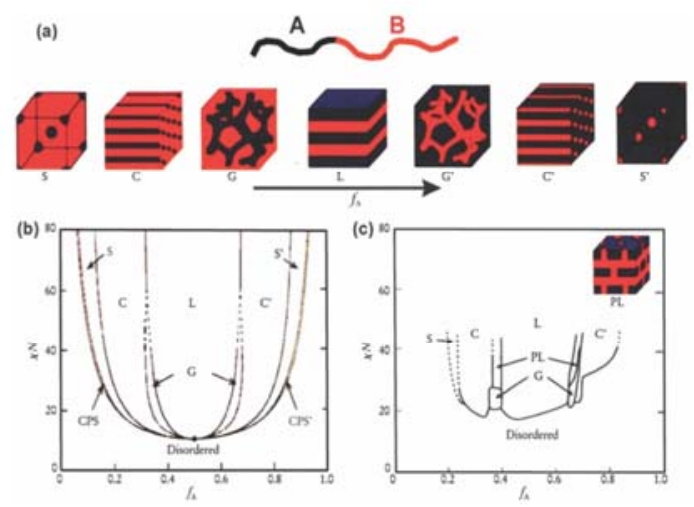

Fig. 1. (a) Morphologies at equilibrium of $A B$ diblock copolymers in bulk as function of the $A$ blocks volume fraction $\left(f_{A}\right)$. S and S' are body-centered-cubic spheres, $\mathrm{C}$ and $\mathrm{C}$ ' are hexagonally packed cylinders, G and G' are bicontinuous gyroids, and L is the lamellar morphology. (b) Selfconsistent mean-field theory phase diagram of an AB diblocks; with CPS and CPS' closely packed spheres. (c) 
Experimental phase diagram of polyisoprene-block-polystyrene diblock copolymers, in which $f_{A}$ represents the volume fraction of polyisoprene; with perforated lamellae, PL. Reproduced with permission from reference [10] and [1], for reference [1] with the permission of the American Institute of Physics.

Self-assembled BCPs can then combine and concentrate properties in controlled nanomorphologies, which present a double interest. (i) The first one is to optimize the global efficiency of the electrolyte materials, and (ii) the second one is to use BCPs as model systems to gain knowledge and ultimately develop more efficient electrolyte materials. Thus, in order to control and improve the polyelectrolyte performances it is essential to characterize the morphologies at the nanometer length scale and pointing out the structure / properties relationship.

Microscopy, with transmission electron microscopy (TEM) and atomic force microscopy (AFM) are methods of choice for the structural characterisation. Microscopy approach has the advantage of a direct visualization of the morphology and the disadvantage of a limited representativeness of the collected pictures. To complement microscopy, scattering techniques such as SAXS and SANS, are frequently performed. Indeed, both SAXS and SANS permit to collect structural averaged data in a large volume of sample; the difficulty is the need of mathematical models to transfer the data from the reciprocal space to the real space.[11] Also, SAXS and SANS are well complementary to each other; for instance, the high brilliance of the X-ray sources allows a fast data acquisition (below the second) together with a high resolution which is essential to investigate fast structural kinetic; on the other hand, neutron contrast variation approach allows to selectively enhance or match the contrast in multiphasic systems, which is essential for a detailed description of complex morphologies. Indeed, the neutron coherent scattering length of an atom gives its scattering efficiency and depends on its nucleus composition. Nevertheless, two isotopes of the same element present different scattering lengths; consequently, isotope exchange can be performed in a material to tune selectively the coherent scattering length densities (SLD) of one phase, while preserving the structural, physical and chemical properties. This substitution is regularly used between hydrogen and deuterium, leading for example in the case of water to SLDs of -0.56 and $6.39 \times 10^{10} \mathrm{~cm}^{-2}$ for $\mathrm{H}_{2} \mathrm{O}$ and $\mathrm{D}_{2} \mathrm{O}$ respectively. The wide range of SLDs allows then mixtures of $\mathrm{H}_{2} \mathrm{O}-\mathrm{D}_{2} \mathrm{O}$ to match the majority of polymers SLD's. In this paper, the scattering curves are plotted as a function of the scattering vector $q$ defined as followed: $q=4 \pi / \lambda \sin (\theta / 2)$ with $\theta$ the scattering angle. Performing small angle scattering on phase segregated BCPs bulk samples usually permit to discriminate the different morphologies thanks to the relative structural peak $q$-positions and permit also to determine the lattice parameters.[12] More details on this particular point are given in annex A.

The present paper points out the essential input of scattering, and especially SANS, in the morphology characterization of fuel cell ionomer membranes and battery polymer electrolytes made of BCPs. Nevertheless, this paper does not aim to be an exhaustive review on fuel cell or battery materials, neither on BCPs but will give an overview based on selected published papers. Finally, few useful analytical tools and models are presented all along the paragraphs and detailed in annex.

\section{BCP ionomer membranes for fuel cell}

Ionomer membranes are currently employed as a separator in prototype and commercialized proton exchange membrane fuel cells (PEMFCs) running at low temperature, targeting a vast range of applications: from small portable to large stationary 
power sources. The basic principle of hydrogen fuel cell is schematized on figure 2. (i) Hydrogen is decomposed in protons and electrons on the anode side, then (ii) the protons are transported through the hydrated membrane to the cathode, (iii) to finally produce water after recombination with the oxygen from air and electrons transported through the external electrical circuit. The ionomer membrane plays a central role and as a consequence it is a key element toward PEMFC performances improvement. The membrane must be chemically and electrochemically stable, have good mechanical strength, have high proton conductivity and must be synthesized at low cost. In the last few decades, a significant number of ionomers have been synthesized to meet these requirements, such as sulfonated aromatic hydrocarbon polymers, e.g. polyimides [13], poly(ether ether ketone) [14], poly(phenylene) [15], poly(phenylene sulfone) [16], or polybenzimidazole [17]. Nonetheless, the membranes of reference, in terms of durability and performance, remains perfluoro-sulfonated membranes, of which Nafion commercialized in the 60's by DuPont de Nemours is the first example. More precisely, Nafion polymer is made of polytetrafluoroethylene backbone with perfluorinated side chains each ended by a sulfonic acid function. However, these fluorinated ionomer membranes are still expensive to produce, due to a complex synthesis. In addition, they are not suitable for high-temperature PEMFC applications (typically above $130{ }^{\circ} \mathrm{C}$ ) because of the drop of proton conductivity above 100 ${ }^{\circ} \mathrm{C}$, correlated with the membrane drying. Also, Nafion membranes are not good candidates for direct methanol fuel cells (DMFC) due to their low methanol crossover resistance. Therefore, the synthesis of new alternative ionomer materials so as their structural and diffusion characterization are still necessary. For that purpose, neutron and X-ray scattering techniques are particularly well-suited tools. $[18,19]$

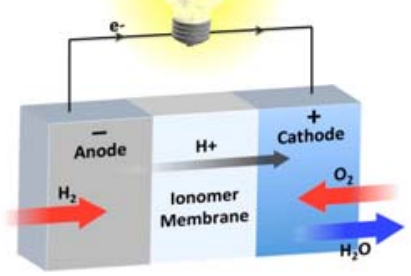

Fig. 2. Schematic representation of proton exchange membrane fuel cell principle.

Nafion remains also a major reference among the ionomer membranes in term of morphology. Indeed, it was intensively investigated by SAXS and SANS, to point out the role of morphology on the remarkable macroscopic properties. Nonetheless, the studies were leading to contradictory structural conclusions, mainly as a matter of fact of the delicate interpretation of scattering data. The Nafion typical scattering curve ( $c f$. figure $3)[20]$ presents peaks at large angles corresponding to the amorphous and crystalline peaks, signature of the macromolecular chains arrangement. The well-known ionomer peak positioned at a scattering vector $q$ about $0.15 \AA^{-1}$ and systematically observed on ionomer material is correlated to the segregation between the hydrated ion rich and the hydrophobic polymer phases. In the intermediate $q$-range a large bump named the matrix-knee, commonly correlated to the crystallinity is sited on a general $q^{-1}$ slope. Finally, in the ultra- 
small angles region, i.e. below $0.005 \AA^{-1}$, an upturn associated to large length scale morphologies was observed and analyzed with Debye-Bueche [21] and Voronoï [22] models.[20, 23]

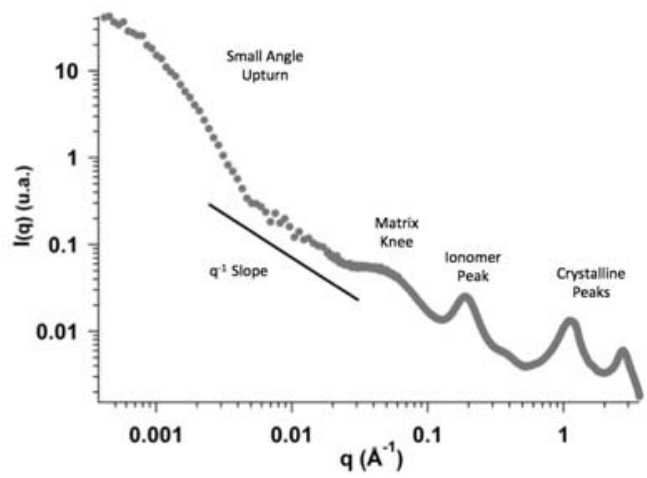

Fig. 3. Wide, Small and Ultra Small Angles X-ray Scattering data (respectively WAXS, SAXS and USAXS) measured on a hydrated Nafion membrane. Figure reproduced with permission from reference [20].

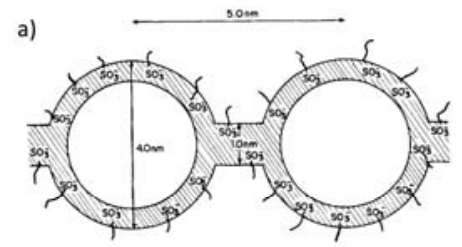

b)

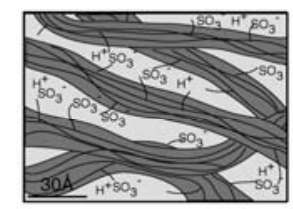

c)

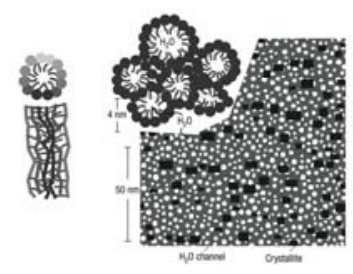

Fig. 4. (a) The Gierke's cluster-network model.[24-26] (b) The direct cylinder model.[23, 27] (c) The Schmidt-Rohr et al. inverted cylinder model.[28] Figures reproduced and modified with permission from references $[18,26,28]$.

The pioneer Nafion structural model was the Gierke's cluster-network model, [24, 25] with spherical clusters of sulfonic acid groups (i.e. inverted micelles), connected by narrow channels within an amorphous fluorocarbon matrix ( $c f$. figure 4a). In 2002, a direct fibrillar model was proposed, considering the aggregation of the fluorocarbon chains into elongated objects surrounded by the ionic groups and water. This model is based on SAXS and SANS data collected at much smaller $q$ compare to Gierke's model, i.e. information on the larger 
dimension ( $c f$. figure $4 \mathrm{~b}$ ) and also over a wide range of water content.[23, 27] The systematic presence of a $q^{-1}$ slope at small $q$ was a key clue, suggesting elongated scattering objects. In addition, this model is able to describe the orientation of the structure under an uniaxial stretching (cf. figure 5).[20, 29] In 2008, an inverted cylinder model was proposed based on new analysis of existing SAXS curves using numerical Fourier transform techniques.[28] This model describes Nafion as parallel cylinders decorated inside with ionic groups and water within a fluorocarbon matrix ( $c f$. figure $4 c)$. More recently, Nafion was described by locally flat and narrow water domains (about $1 \mathrm{~nm}$ ) in a fluorocarbon matrix.[30] To conclude, all those models agree at least on one point, there is a cocontinuous phase separation between the hydrophobic fluorinated polymer and the hydrated ionic group phase at the nanometer length scale, even if they disagree on the exact shape of those phases.
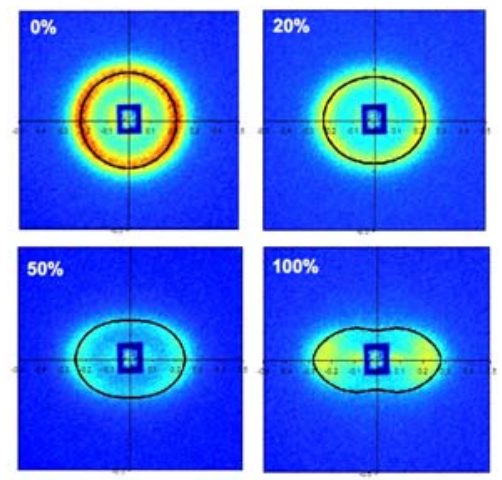

Fig. 5. SANS experimental results collected on a drawn Nafion film, with deformation $0,20,50$ and $100 \%$. The black line corresponds to the ionomer peak position calculated through the cylinder deformation model[29] with the deformation ratio as a single adjustable parameter. Figure extracted with permission from reference [20].

SANS with contrast variation is a powerful method to investigate the nano-morphology of Nafion and other ionomer membranes.[18] Indeed, the structures are extremely dependent on water content and in most cases they are observed hydrated such as fuel cell running conditions. Then fully or partially deuterated water $\left(\mathrm{H}_{2} \mathrm{O}-\mathrm{D}_{2} \mathrm{O}\right.$ mixtures $)$ can be used for contrast matching or enhancing. To illustrate this point, on figure 6 are presented SANS curves collected on the same Nafion membrane highly hydrated with four different $\mathrm{H}_{2} \mathrm{O}$ $\mathrm{D}_{2} \mathrm{O}$ mixtures. The impact on the scattering curves is dramatic and can be predicted with a core-shell cylinder form factor assuming that the scattered intensity can be expressed as the product of a structure factor $S(q)$ and a form factor $P(q):[31]$

$$
I(q)=K \cdot S(q) \cdot P(q)
$$

where $K$ is a scaling factor related to the number of scattering objects. The form factor describes the specific shape of the scattering objects, and the structure factor their spatial ordering. More conditions on the validity of this expression are discussed in the next paragraph. Here, it is assumed also that the structure factor can be neglected due to the large hydration of the membrane (i.e. polymer volume fraction is $16 \%$ ). The solid black lines on figure 6 correspond to the core-shell cylinder form factor using identical structural 
parameters for the four curves while adjusting the SLDs of the water phase and the hydrated ionic corona.

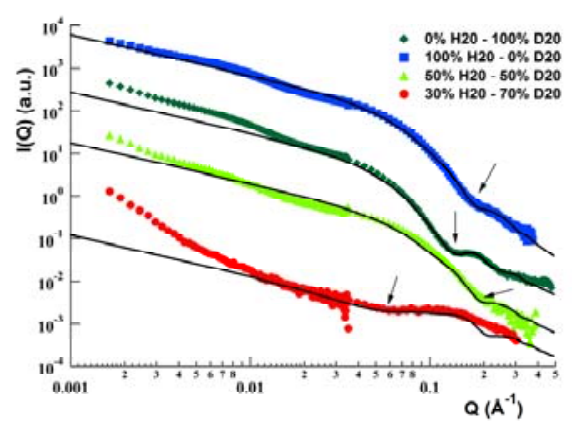

Fig. 6. Contrast variation SANS curves measured on a single swollen Nafion membrane exchanged with $\mathrm{N}\left(\mathrm{CH}_{3}\right)_{4}$ counter ion at four different $\mathrm{H}_{2} \mathrm{O}-\mathrm{D}_{2} \mathrm{O}$ ratios. The membrane polymer volume fraction is $16 \%$. The solid black curves correspond to a core-shell cylinder form factor fits taking into account the contrast variation from the solvent. The four arrows indicate the position of the first minimum for each curve; this minimum is related to the counterions shell interferences. Figure reproduced with permission from reference [27]. Copyright (2002) American Chemical Society.

\section{BCP ionomer membranes}

The design and synthesis of model polymers, such as BCP, is a key element of any research program aimed at improving ionomer membranes. It is expected that properties, such as proton conductivity, is influenced by many factors including the chemical structure (i.e. composition and nature of the polymer chain), ion exchange capacity (IEC), water content, and also by the multi-length-scale morphology. The study of model materials can then provides valuable knowledge on proton transport mechanisms and ultimately will lead to the design of future generation of membranes. Several non-fluorinated copolymers containing a partially sulfonated polystyrene block had been synthesized, such as sulfonated poly(styrene- $b$-[ethylene-co-butylene]- $b$-styrene),[32-37] sulfonated poly(styrene- $b$-isobutylene- $b$-styrene), $[38,39]$ sulfonated hydrogenated poly(butadiene- $b$ styrene),[40, 41] sulfonated poly(styrene- $b$ - [ethylene-co-propylene]),[42, 43] sulfonated poly(styrene- $b$-ethylene/propylene- $b$-styrene),[42] block copolymers sulfonated poly(styrene- $b$-methylbutylene).[44] In most cases, the structural investigations on ionomer BCPs reveal hierarchical structures. Indeed, the regular BCPs self-assembly occurs at the length scale of few tens of nanometers and the charges clustering arises within the ionomer phase at the length scale of few nanometers; that was demonstrated by Weiss and coworkers by small angle scattering.[34]

In order to take advantage of the chemical stability of fluorinated polymers, BCPs such as sulfonated poly-([vinylidene difluoride-co-hexafluoropropylene]-b-styrene) block copolymers,[45-47] were also explored. 

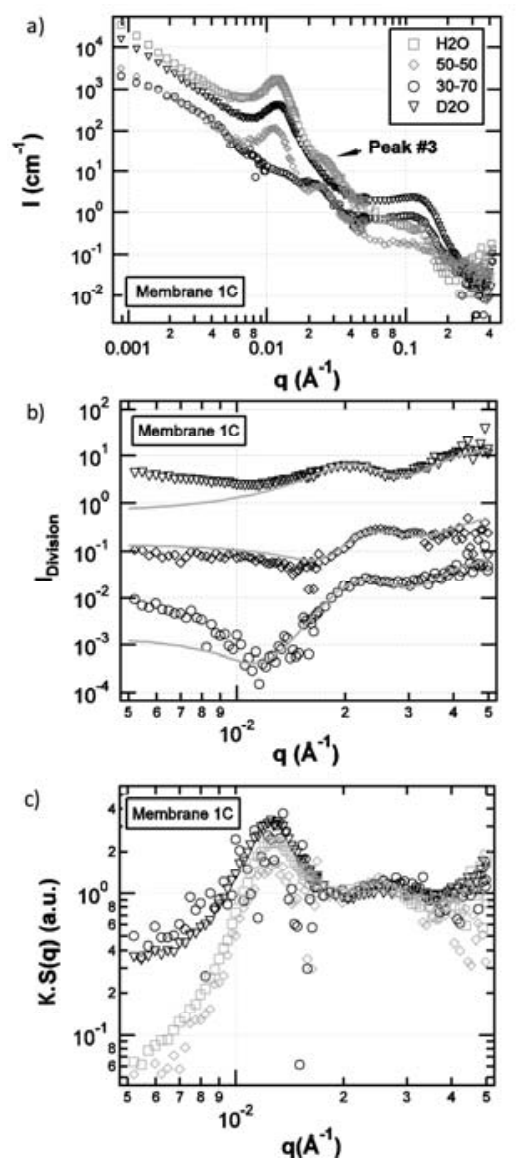

Fig. 7. (a) SANS spectra collected on one film swelled with four different $\mathrm{H}_{2} \mathrm{O}-\mathrm{D}_{2} \mathrm{O}$ mixtures: 100-0 (squares), 50-50 (diamonds), 30-70 (circles) and 0-100 (triangles). (b) SANS spectra divided by the $\mathrm{H}_{2} \mathrm{O}$ spectra: $\mathrm{D}_{2} \mathrm{O} / \mathrm{H}_{2} \mathrm{O}$ (triangles), $30-70 / \mathrm{H}_{2} \mathrm{O}$ (circles) and $50-50 / \mathrm{H}_{2} \mathrm{O}$ (diamonds). The solid lines represent the theoretical results for the normalized spectra $P_{d i v}$. For the sake of clarity, the curves are offset. The fits are performed in absolute intensity. (c) Calculated structure factor, $K . S(q)$, for the film swollen in $\mathrm{H}_{2} \mathrm{O}$ (square), 50-50 (diamonds), 30-70 (circles) and $\mathrm{D}_{2} \mathrm{O}$ (triangles). Figures reproduced with permission from reference [45]. Copyright (2006) American Chemical Society.

Holdcroft and coworkers have synthetized original block copolymers ionomer with fluorinated poly (vinylidene difluoride-co-hexafluoropropylene) and sulfonated polystyrene ionomer blocks.[46-48] The blocks incompatibility and low dispersion lead to well-defined structures as revealed by TEM imaging and SANS over a wide range of length scales while varying blocks dimension and ion exchange capacity (IEC).[45] Taking advantage of the SANS contrast variation, the films were soaked in four different $\mathrm{H}_{2} \mathrm{O}-\mathrm{D}_{2} \mathrm{O}$ mixtures: 100-0, 50-50, 30-70 and 0-100. These mixtures present SLDs of -0.56, 2.915, 4.305, and $6.39 \times$ $10^{10} \mathrm{~cm}^{-2}$, respectively. On the other hand, the polystyrene has a SLD of $1.41 \times 10^{10} \mathrm{~cm}^{-2}$ and the fluoropolymer $3.22 \times 10^{10} \mathrm{~cm}^{-2} ;$ [45] in consequence no contrast matching was expected. To analyze the large length scale SANS data ( $c f$. figure 7a), it was assumed that the scattered intensity can be expressed as the product of a structure factor $S(q)$ and a form factor $P(q)$ ( $c f$. equation 2). It is important to note that Equation 2 is valid in two cases: (i) the scattering objects are centro-symmetric (typically spheres) or (ii) the scattering objects are anisotropic (e.g. cylinders, disks) but locally oriented.[49] Also the expression is valid 
in the $q$-range where q. $L>1$, with $L$ the characteristic dimension of the structure. In addition, it is assumed that changing the solvent $\mathrm{H}_{2} \mathrm{O}-\mathrm{D}_{2} \mathrm{O}$ ratio has no effect on the morphology,[50] i.e. the structure factor is expected to be invariant at all contrast. Finally, it is assumed that the geometry of the scattering objects remains the same but the SANS form factor is expected to vary due to the variation of the SLDs. Under all these assumptions, if two spectra collected on the same membrane but soaked in two different solvents are divided by each other, then the $S(q)$ and $K$ components will be eliminated, resulting to a ratio of two form factors ( $c f$. equation 3 ). Then this ratio can be compared to curves calculated for different object shapes.[23, 51] Experimentally, the spectra collected on a membrane soaked in 50-50, 30-70, and $\mathrm{D}_{2} \mathrm{O}$ mixtures (solvent1) were divided by that soaked in $\mathrm{H}_{2} \mathrm{O}$ (solvent2) to obtain the $P_{d i v}(q)$.

$$
I_{\text {div }}(q)=\frac{I_{\text {solvent } 1}(q)}{I_{\text {solvent } 2}(q)}=\frac{K \cdot S(q) \cdot P_{\text {solvent } 1}(q)}{K \cdot S(q) \cdot P_{\text {solvent } 2}(q)}=P_{\text {div }}(q)
$$

Finally, once the theoretical $P(q)$ are determined, then the products $K . S(q)$ are calculated by dividing the measured spectra by the corresponding $P(q)$ as expressed below for solvent 1 :

$$
K . S(q)=I_{\text {solvent } 1}(q) / P_{\text {solvent } 1}(q)
$$

where $K$ and $S(q)$ are both contrast independent, then all the $K . S(q)$, calculated from the different solvents, should all collapse on each other. Figure $7 \mathrm{~b}$ presents the results for divided experimental spectra. The fact that the divisions are not flat means that the scattering objects cannot be modeled as a basic two-phases system, but at least three phases. This first observation is a motivation to consider core-shell form factors. Then the shape of the scattering object has to be determined; spherical and cylindrical core-shell form factors gave rise to unrealistic parameters or low fit quality, only the core-shell discoidal form factor led to a reasonable analysis ( $c f$. figure $7 b$ ). Indeed, each scattering object is considered as a disk-like core with an external layer (shell) on both sides. The core is defined by its radius $r$, thickness $t$, thickness polydispersity $\sigma$. The shell is defined by its thickness, $s$. The SLDs were calculated considering a core made of hydrated sulfonated PS, a pure PS shell, and a surrounding matrix of fluoropolymer. In that case the unknown fitting parameters are $\phi_{p}$, the polymer volume fraction of the disk core, $t$, the core thickness with $\sigma$ its polydispersity, and $s$, the shell thickness. The same set of structural parameters was used to fit the three normalized spectra collected on the same samples, only the SLD of the swollen core was varying and calculated with the proper solvent's SLD. Curves showing the fits are presented in figure $7 \mathrm{~b}$. The fit approach is good enough to confirm the core-shell disk-like form factor, considering that most of the parameters are strongly constrained and considering also the fit agreement in relative intensity for the three curves simultaneously. Finally, the $K . S(q)$ were calculated, the results are presented in figure $7 \mathrm{c}$, with a first peak at $q^{*}$ and second order at $2 q^{*}$ consistent with a lamellar ordering of the disk-like objects. All four $K . S(q)$ collapse on each other which supports the analysis assumptions. To conclude the SANS contrast variation analysis was a key element in this study, pointing out the precise hierarchical structure brought by the block copolymer selfassembly and the sulfonic acid groups clustering in the sPS phase. As shown on figure 8, the fine SANS analysis points out the presence of a thin dead zone, in term of conductivity, of pure PS in-between the sPS and the fluorinated lamellas. 


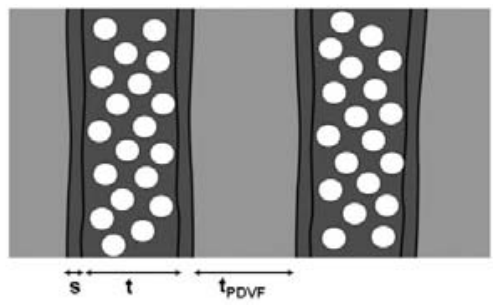

Fig. 8. Sketch of the membrane structure. The lighter gray corresponds to the fluorous lamellae with a thickness $t_{\mathrm{PVDF}}$, and the darker gray corresponds to the PS lamellae. Within the PS lamellae there are two different regions: the central region contains hydrated sulfonated polystyrene, with a thickness $t$; at the interfaces are located thin layers of pure polystyrene, with a thickness $s$. The white solid circles within the sulfonated polystyrene domains represent hydrated ionic aggregates. Figure reproduced with permission from reference [45]. Copyright (2006) American Chemical Society.

A similar SANS approach was performed on grafted block copolymers perfluorinated ionomer membranes made of partially sulfonated poly-([vinylidene difuoride-cochlorotriuoroethylene]-g-styrene) on which the grafting densities and the polystyrene side chains length and sulfonation rate was modulated.[52] X-ray scattering at large and small angles permitted to collect data on crystallinity and nano-morphology on the non-hydrated films. To complete the study a SANS experiment with contrast variation was performed on hydrated samples using four different $\mathrm{H}_{2} \mathrm{O}-\mathrm{D}_{2} \mathrm{O}$ mixtures, i.e. 100-0, 70-30, 40-60 and 0100.[53] The respective SLDs are $-0.56,1.52,2.91$, and $6.37 \times 10^{10} \mathrm{~cm}^{-2}$. The fluorinated polymer SLD was estimated at $2.87 \times 10^{10} \mathrm{~cm}^{-2}$ and the polystyrene one $1.41 \times 10^{10} \mathrm{~cm}^{-2}$; consequently, contrast matching can be expected. This SANS experiment allowed quantifying (i) the swelling of the polymer films, (ii) the size and order of the ion-rich regions, and (iii) the size and order of the fluorinated domains. Figure 9a presents the scattering curves and reveal two broad peaks located within two $q$-ranges 0.04 to $0.06 \AA^{-1}$ and 0.1 to $0.3 \AA^{-1}$. Those peaks are analyzed as signatures of a hierarchical morphology consisting of a disordered and partially phase-separated system, with two characteristic features and dimensions ( $c f$. figure $9 \mathrm{~b}$ ). Thanks to contrast variation and matching both peaks were attributed to a structure consisting of fluorinated domains in a partially sulfonated PS matrix at large length scale and ion-rich domains within the PS matrix at smaller length scale. Practically, the SANS data were interpreted by fitting the Kinning \& Thomas model [54] ( $c f$. annex B) to the fluorinated peak and the Teubner \& Strey model [55] ( $c f$. annex C) to the peak associated to the ion-rich domains. This combination of models provided the best fits to the data with a reasonable number of parameters. The analysis of the structural parameters permitted to point out the impact of the morphology on water uptake and proton conductivity. It was found that the structural swelling observed at the nanometer length scale was consistent with the membrane water uptake, indicating consistency between microscopic and macroscopic length scale swellings in these materials. Also, it was demonstrated that phase separation between fluorinated polymer and PS phase is limited because of the competition with the sulfonated acid groups aggregation mechanism. The similarities in water uptake and conductivity values for membranes of radically different morphology were also pointed out, particularly when volume-based quantities from references [45] and [53] are compared. To summarize, the present SANS study points out that the control of water uptake through the macromolecular structure is a key feature in designing efficient materials. 


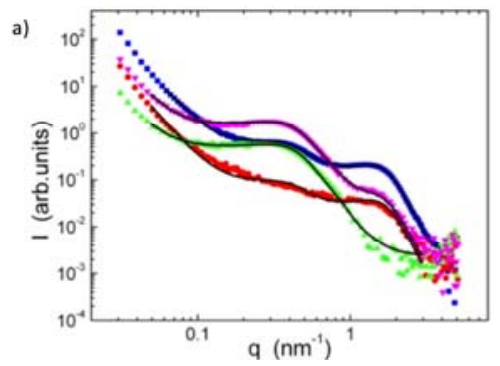

b)

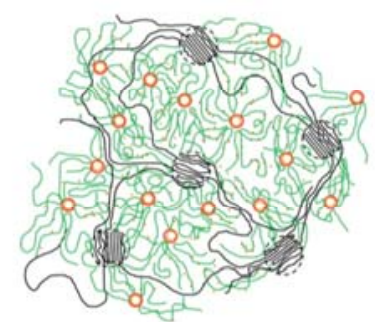

Fig. 9. (a) SANS spectra measured on an ionomer film with an IEC of $2 \mathrm{mmol} / \mathrm{g}$ swollen in four $\mathrm{H}_{2} \mathrm{O}-\mathrm{D}_{2} \mathrm{O}$ mixtures: $\mathrm{D}_{2} \mathrm{O}$ (squares), $\mathrm{H}_{2} \mathrm{O}$ (downward triangles), 40-60 (circles), and 70-30 (upward triangles). The water content was $36 \%$. The solid black lines correspond to the fits using the combined models: Kinning \& Thomas and Teubner \& Strey model at small and larger $q$ respectively. Data sets have been shifted vertically for sake of clarity. (b) Schematic representation of the structural model used to depict the morphology and fit the SANS data. In black the fluorinated backbones, with PVDF rich spherical domains, dispersed in a continuous PS matrix (green). At smaller length scales, the ion-rich domains in red. Figures reproduced with permission from reference [53]. Copyright (2013) American Chemical Society.

Finally, to illustrate the structure / properties relationship using model ionomer diblock copolymers, we are now focussing on a study performed on partially sulfonated poly(styrene- $b$-methyl methacrylate) (PS- $b$-PMMA) that point out the role of controlled morphologies on the proton conductivity and the water uptake.[56] The advantage of this system is that series of polymers presenting similar sulfonated PS blocks were used while varying the PMMA block dimension. The structure and properties of the sulfonated PS (sPS) phase are supposed to be identical. By combining TEM and SANS the different morphologies were described. Depending on the molecular weight of the copolymers and the degree of sulfonation of the PS block, disordered (DIS), lamellar (LAM), hexagonal cylindrical packing (HEX) and hexagonally perforated lamellae (HPL) phases were observed. The swelling of the films exposed to water was significantly different according to the morphology. For instance, the samples exhibiting a lamellar structure undergo a consequent increase in the inter-lamellar dimension, whereas no swelling was observed for the samples presenting the HEX or HPL morphologies. It is worth to note that for those morphologies the matrix is made of PS. As expected, the water uptake of the films is accompanied by a significant increase in proton conductivity. Nevertheless, this increase was also dependent on the nano-metric morphology. In order to highlight the role of structure, the authors have compared the conductivities normalized by the volume fractions of the conductive phase (i.e. the domains formed by sPS, PS, and water). Figure 10 presents the normalized conductivity which increases according to the following sequence of morphologies: DIS, HEX, HPL and LAM, in agreement with hopping, 1D and 2D charges transport mechanisms; i.e. hopping (DIS), 1D (HEX) and 2D (HPL and LAM). 


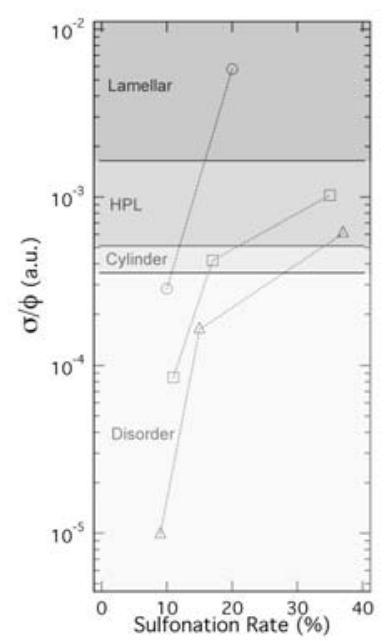

Fig. 10. Normalized proton conductivity $(\sigma / \Phi)$ as a function of the sulfonation rate for the three series of diblock copolymers varying the ratio of PS (circles $53 \%$, squares $22 \%$ and triangles $15 \%$ ). Here $\Phi$ refers to the volume fraction of the conducting phase, i.e. PS + sPS + water domains. Figure reproduced with permission from reference [56]. Copyright (2008) American Chemical Society.

\section{BCP solid polyelectrolytes for battery}

Polymer electrolytes can be used as separator between the electrodes of secondary batteries. The two principal expected properties are: the ability to dissociate the salt and also to efficiently transport the ions during charge and discharge. The basic secondary battery principle is schematized in figure 11. During the charge, the lithium ions migrate through the electrolyte toward the anode and get intercalated or inserted in the electrode material (typically carbon graphite or lithium metal). During the discharge, the lithium ions diffusion toward the cathode, where they get inserted in the electrode material (typically cobalt oxide), is accompanied by an electron current in the external circuit.

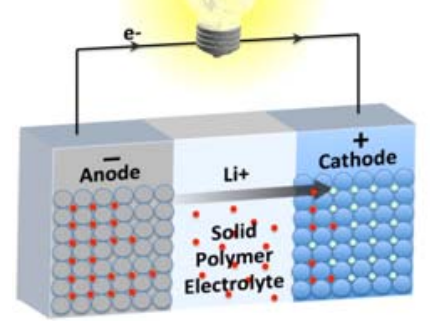

Fig. 11. Schematic representation of lithium solid polymer electrolyte battery principle during discharge.

Most of the batteries currently commercialized are prepared with electrolyte containing solvents, such as ethylene or propylene carbonate. Those solvents facilitate the ion transport; but unfortunately, they may cause critical safety issues: chemical leakage and risk of flammability. Nowadays, this is major concern in the development of secure 
batteries for nomad and transportation applications. A suitable alternative to solvent containing electrolyte, is solid polymer electrolyte (SPE), despite poorer ion conductivity; indeed, important efforts are done to reach the objective of $10^{-3}$ to $10^{-2} \mathrm{~S}_{\mathrm{cm}} \mathrm{cm}^{-1}$ at working temperature. In addition, it is expected that the high mechanical modulus of the solid polyelectrolytes $(>6 \mathrm{GPa}$ ) prevent the formation of dendrites on lithium anode during the charge.[57] Another issue that polymer electrolytes can overcome is the anions concentration gradient between the electrodes occurring during discharge and limiting the maximum current accessible; only a fraction of the current is carried by the lithium ions. This current fraction can be measured and expressed in term of transport number, $t_{+}$, which can range from 0 to 1 . In PEO blended with lithium salt, $t_{+}$is ranging generally between 0.1 and $0.2,[58]$ meaning an extremely low fraction of the current is carried by the lithium ions. Recently, single ion polymers were synthesized, with anions grafted on the polymer chain, leading to a significant increase of the transport number toward the unity.[59, 60]

The classical SPE polymers are poly(ethylene oxyde) (PEO), poly(vinylidene fluoride) (PVDF) or polycarbonates based polymer presenting similar chemical function than the carbonate solvents, such as polytrimethylene carbonate.[61] On figure 12 extracted from reference [62] are presented the ionic conductivity versus temperature of different polymer based electrolytes. The most efficient (highest conductivity at lowest temperature) are the organic electrolyte (containing solvent), then the gel electrolyte (solid containing solvent), then the SPE containing free lithium salts. The poorer performances are for single ion conductor[59, 60] and solid lithium phosphorous oxynitride electrolyte LiPON.[63]

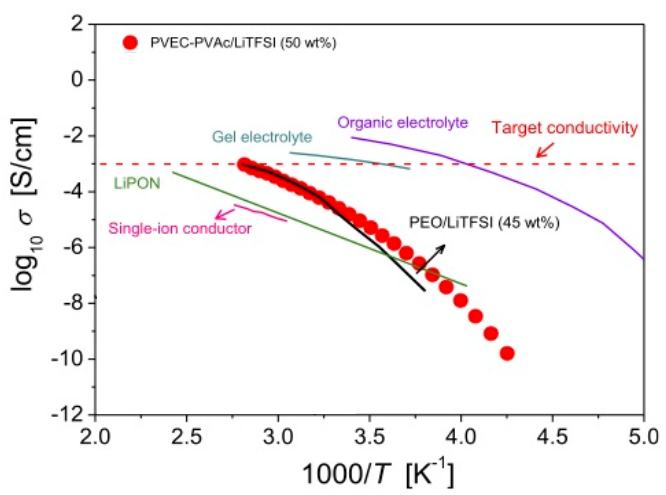

Fig. 12. Ionic conductivity versus temperature curves for selected representative lithium based electrolytes: PVEC-PVAc/LiTFSI (50 wt\%) block copolymer,[62] a gel electrolyte made of $1 \mathrm{M}$ $\mathrm{LiPF}_{6} / \mathrm{EC}-\mathrm{PC}(50: 50 \mathrm{vol} \%)$ in $10 \mathrm{wt} \%$ of polyvinylidene difluoride-hexafluoropropylene,[64] an organic electrolyte made of $1 \mathrm{M} \mathrm{LiPF}_{6} / \mathrm{EC}-\mathrm{PC}(50: 50 \mathrm{vol} \%),[65]$ a single-ion electrolyte made of P(STFSILi)- $b$-PEO-b-P(STFSILi),[60] and a lithium phosphorous oxynitride electrolyte (LiPON). Figure extracted and modified with permission from reference [62].

So far, PEO is the most extensively investigated polymer because of its high dielectric constant and its strong lithium ion $\left(\mathrm{Li}^{+}\right)$solvating ability, brought by the $\mathrm{Li}^{+}$coordination on the ether oxygen. Actually, each $\mathrm{Li}^{+}$is potentially coordinating several ether oxygen atoms (on the same or different polymer chains) thus creating transient cross-linking points decreasing the segmental relaxation of the polymer chains. Also, the charges transport involves the segmental motion of the polymer chain and the re-coordination of the ions with neighboring ether oxygen (EO). Meaning that the ratio of EO units per lithium ion is a major parameter (EO:Li), so as the polymer glass transition temperature, $\mathrm{Tg}$, which should be low to facilitate the charges transport $\left(\right.$ e.g. PEO $\left.T g=-60^{\circ} \mathrm{C}\right)$. PEO is a semi-crystalline 
polymer with a melting temperature at the vicinity of $60{ }^{\circ} \mathrm{C}$. The crystallites preserve the mechanical strength of the low $T g$ polyelectrolyte and simultaneity tend to lower the ionic transport, as the conducting phase is quasi exclusively in the amorphous PEO / lithium phase.[66] Nevertheless, it was shown that PEO blended with lithium salt can present crystalline PEO / lithium complex phases,[67] including the $\mathrm{PEO}_{6}: \mathrm{LiAsF}_{6}$ phase that potentially present an enhanced conductivity.[68] In this particular complex phase, Bruce and coworkers claim that the charges diffuse in nano-channels of crystalline PEO, on the other hand the counter ion are located outside the channel and are partially immobile; thus, ameliorating the electrolyte transport number, $t_{+}$. A detailed structural and dynamical study, using SANS and quasi elastic neutron scattering (QENS) was performed by Maranas and coworker on $\mathrm{PEO} / \mathrm{LiClO}_{4}$ as function of temperature and ratio $\mathrm{EO}: \mathrm{Li}$ to investigate further the structure / dynamics relationship.[69] The analysis of the SANS data, taking advantage of the SLD contrast resulting from crystallization, indicates that the $(\mathrm{PEO})_{3}: \mathrm{LiClO}_{4}$ phase presents a cylindrical morphology, with a radius of $125 \AA$ and a length of $700 \AA$. Implying that multiple $(\mathrm{PEO})_{3}: \mathrm{LiClO}_{4}$ helices align to form those large cylindrical structures. The authors have also localized the $\mathrm{LiClO}_{4}$ nearby the amorphous domains, inducing a SLD contrast between the amorphous $\mathrm{PEO} / \mathrm{LiClO}_{4}$ bilayer and the crystalline lamellae detectable by SANS. The dimensions of the crystalline lamellae and the amorphous bilayer are both maximized at a particular salt concentration (i.e. 14:1) so as ion conductivity, despite the fact that this sample is partially crystalline. Nonetheless, the most prevailing view is that PEO crystallization is detrimental to the ion diffusion, because of structural tortuosity and chain dynamics limitation (tethered chains confinement). [66] Inelastic neutron scattering (e.g. QENS) is a technique of choice for dynamical investigations; nevertheless, as the incoherent scattering cross-section of lithium is negligible compared to hydrogen, inelastic neutron scattering is quasi exclusively sensitive to the dynamics of the hydrogenous polyelectrolyte chains and not to the mobile $\mathrm{Li}^{+}$.[70] This technique is then complementary to dielectric or impedance spectroscopy in which the signal is dominated by the dynamics of the $\mathrm{Li}^{+}$. The correlation between the segmental relaxation in polymer electrolytes and the dynamics of the ionic charges, at the nanosecond and picosecond time scale, is extremely informative: the general trend is that the $\mathrm{Li}^{+}$ions aggregate with multiple polymer chains and slow down the molecular and ionic mobility, i.e. creation of transient cross-links as mention previously.

\section{BCP solid polyelectrolytes}

In the perspective of developing solid polyelectrolytes, BCPs present the major advantage of fully decoupling ion conduction and mechanical properties. In addition, as already mentioned, BCPs electrolytes can be exploited as a model system to investigate the structure / properties relationship. Recently, Bouchet and coworkers have explored a wide range of block copolymers architectures made of polystyrene (PS) and PEO, i.e. diblock, triblock (ABA \& BAB) and comb-like copolymers.[71] They conclude that the triblock PS$b$-PEO- $b$-PS structure presents the best compromise between ion conductivity and mechanical properties. Also, the salt repartition is a crucial point to understand and improve the diffusion mechanisms. Wang and coworker have demonstrated theoretically that the ion distribution profile, so as the electrostatic potential, depend on the blocks dielectric contrast and also on the capacity of lithium ions to redistribute within the iondissolving phase.[72] In parallel, Bouchet and coworkers have demonstrated the presence of a dead zone - in term of conductivity - at the interphases PS/PEO in PS- $b$-PEO diblock copolymers.[73] This dead zone is constituted by 4 to 5 units of EO and can be explained by the constraint imposed by the PS block limiting the mobility of the PEO and more 
particularly on the first EO units. So far, the BCP solid polyelectrolyte self-assembly was intensively studied by SAXS and microscopy techniques, such as TEM. It is worth to note that in many of those studies the main fundamental objective is the determination of the Flory-Huggins parameter, $\chi$, in order to point out the role of the lithium salt on the selfassembly mechanism.

Nevertheless, one of the pioneer works on BCP solid polyelectrolyte has demonstrated the great potential of SANS in the domain.[74] The system investigated by Ruzette et al. was a poly (methyl methacrylate - $b$ - oligo oxyethylene methacrylate) BCP blended with $\mathrm{LiCF}_{3} \mathrm{SO}_{3}$ salt. The advantage of oligo oxyethylene methacrylate compared to regular PEO is the absence of crystallinity while preserving the same chemical functions. The neat copolymer presents a disordered phase, i.e segmentally mixed at temperatures ranging between 100 and $200{ }^{\circ} \mathrm{C}$. The order to disorder transition temperature, $T_{O D T}$, is below the $\mathrm{Tg}$ of the whole copolymer. The authors have demonstrated that increasing the salt content is raising the $T_{O D T}$ of the block copolymer bringing a microphase separation. Also, there is preferential complexation of $\mathrm{LiCF}_{3} \mathrm{SO}_{3}$ with the ether linkages of POEM compared to the carbonyl groups of methacrylate, inducing a weakening of the segmental interactions.

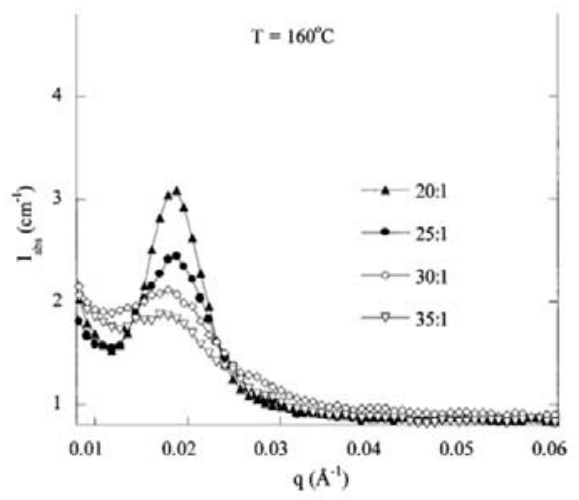

Fig. 13. SANS scattering intensity curves collected on salt-doped PMMA-b-POEM at $160{ }^{\circ} \mathrm{C}$ and four different salt concentrations (EO:Li): (20:1), (25:1), (30:1) and (35:1). Reproduced with permission from reference [74].

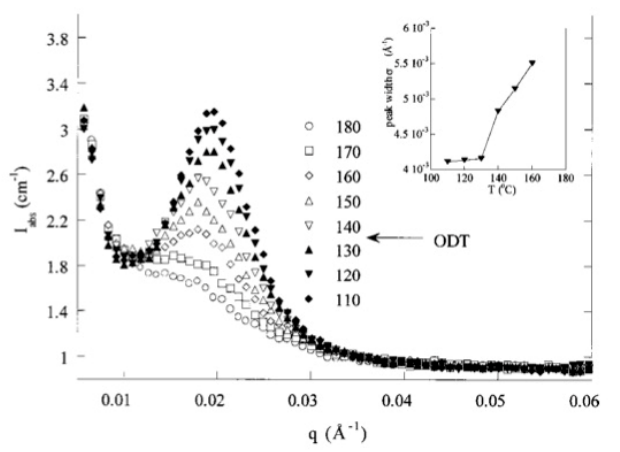

Fig. 14. SANS scattering intensity curves collected on PMMA- $b$-POEM blended with $\mathrm{LiCF}_{3} \mathrm{SO}_{3}(30: 1)$, as a function of temperature. The inset presents the variation in peak width with temperature. Reproduced with permission from reference [74]. 
Figure 13 presents the SANS spectra collected on the BCP with different amount of salt at $160{ }^{\circ} \mathrm{C}$. At higher salt content 20:1 and 25:1 (solid markers) sharp intense scattering maximums are observed, which are the first order Bragg reflections. Those are characteristic of the ordered micro-phase separated state. On the other hand at lower salt content 30:1 and 35:1 (empty markers) broad low intensity scattering maximums are observed, which indicate blocks segmentally mixed characteristic of a correlation hole, first described by Liebler.[2] More classically, temperature was change in the range 110 to 180 ${ }^{\circ} \mathrm{C}$ for a given salt concentrations ( $c f$. figure 14), in the inset the peak width is used to point out the $T_{O D T}$. The results are presented in table 1, and show that the $T_{O D T}$ increases significantly with the salt content. Both extreme systems: (i) below 40:1 are always disordered and (ii) 20:1 are systematically ordered. This result indicates that increasing the salt content raise the Flory-Huggins parameter, $\chi$.

Table 1. Phase behavior and order to disorder transition temperatures, TODT, of salt-doped PMMA$b$-POEM as a function of salt concentration. Reproduced with permission from reference [74].

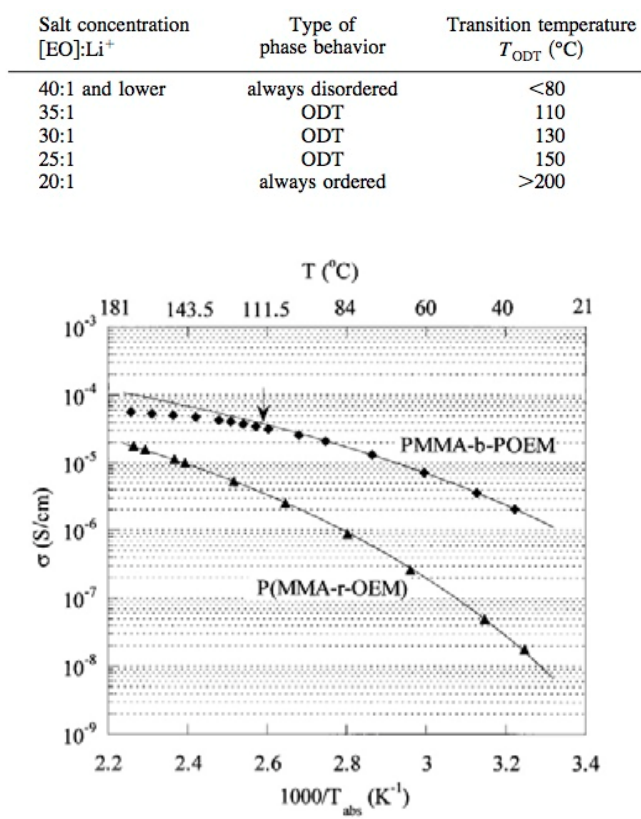

Fig. 15. Ionic conductivity versus temperature measured on salt-doped PMMA-b-POEM and $\mathrm{P}(\mathrm{MMA}-r$-OEM) with a salt concentration of $(30: 1)$. The solid lines correspond to the fits with the Vogel-Tammann-Fulcher (VTF) relation which best fits the non-Arrhenius behaviour of the data. Reproduced with permission from reference [74].

Conclusively, on figure 15, Ruzette et al. compare the BCP conductivity to a random copolymer with an identical composition, i.e. an identical amount of conducting polymer. The ordered BCP presents a significantly higher conductivity especially at lower temperature; also, the conductivities are increasing and converging with temperature. This increasing rate slows-down dramatically for the BCP above the ODT, i.e. $110^{\circ} \mathrm{C}$ (arrow on figure 15). As expected, at the highest temperature, $170{ }^{\circ} \mathrm{C}$, when the block copolymer is no longer microphase separated, its conductivity approaches that of the random copolymer. More recently, Bergfelt and coworkers have used SANS on partially deuterated triblock copolymer of poly[(oligo ethylene glycol) methyl ether methacrylate] (PMMA- $b$ - 
POEGMA- $b$-PMMA) blended with LiTFSI (9:1)(EO:Li).[75] In this system the PMMA block was selectively deuterated. The neutron experiment was performed at temperatures used during battery tests, i.e. $25,60 \& 95{ }^{\circ} \mathrm{C}$. Considering the elevated salt ratio, those temperatures were expected to be below the system $T_{O D T}$, according to Ruzette et al. observations on similar polymers.[74] Nevertheless, SANS spectra revealed block copolymers in a mixed state, with broad and low intensity peaks, which were interpreted as the result of the correlation hole effect.[2] In order to extract the Flory-Huggins interaction parameter, the random phase approximation (RPA, $c f$. annex D) model was used to fit the data. This approach will be detailed in the next section.

As previously mentioned, one of the main fundamental interrogations when considering BCP is the determination of the Flory-Huggins parameter, $\chi$, value. Adding salt changes considerably the interaction between the blocks, as demonstrated by Ruzette et al.; then equation 1 can be adapted, assuming that the ions are dissolved in only one of the phases. The Flory-Huggins parameter $\chi_{\text {eff }}$ for block copolymers blended with salt becomes:

$$
\chi_{e f f}=\frac{A(r)}{T}+B(r)
$$

with $r$ the molar ratio between the ions and the monomers of ion-containing blocks. Experimentally, $\chi_{\text {eff }}$ can been determined from scattering data, considering (i) the structure factor changes in the disordered phase, (ii) the change in the domain spacing of the ordered phases, or also (iii) the shift in the phase boundaries.

Russell and coworkers [76] have fitted the changes in structure factor of the disordered phase using the RPA theory ( $c f$. annex D). Their experimental data was collected by SANS on PMMA-b-PS diblock copolymers blended with $\mathrm{LiCl}$ salt ( $c f$. figures 16 \& 17). Considering the following expression of the Flory-Huggins parameter with an enthalpic, $\chi_{H}$, and entropic components, $\chi_{S}:[76]$

$$
\chi=\frac{\chi_{H}}{T}+\chi_{S}
$$

They have shown that the entropic contribution $\chi_{S}$ for the salt complexed copolymer increases and on the other hand the enthalpic contribution, $\chi_{H}$ decreases, resulting in a nearly temperature-independent $\chi$.

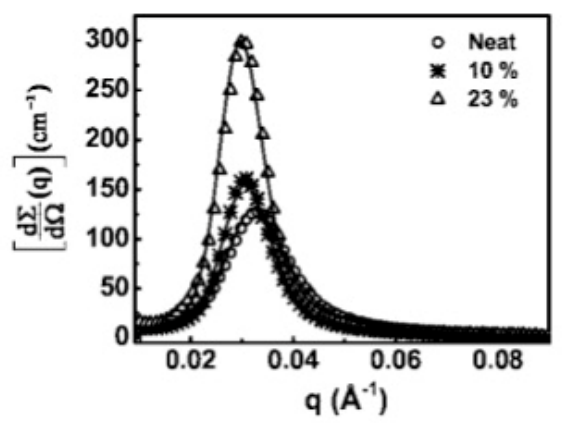

Fig. 16. SANS spectra (symbols) measured at $240{ }^{\circ} \mathrm{C}$ for the neat dPS- $b$-PMMA copolymer and 2 different salt concentrations, $10 \%$, and $23 \%$ of PMMA carbonyl groups coordinated to lithium ions (CO:Li). The solid lines correspond to the calculated profiles with $\chi$ values of $0.03616,0.03647$, and 
0.03702 for the neat copolymer and the copolymers with $10 \%$ and $23 \%$ (CO:Li), respectively. Reproduced with permission from reference [76]. Copyright (2008) American Chemical Society.

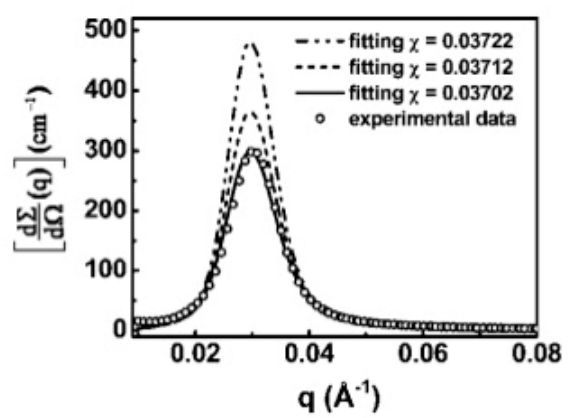

Fig. 17. SANS spectra (open circles) measured at $240{ }^{\circ} \mathrm{C}$ for the dPS- $b$-PMMA copolymer with $23 \%$ (CO:Li). The lines correspond to the calculated profiles with 3 different $\chi$ values, $0.03702,0.03712$, and 0.03722. Reproduced with permission from reference [76]. Copyright (2008) American Chemical Society.

Epps and coworker have exploited the change in the domain spacing, $d^{*}$, in the ordered phases versus salt concentration to estimate $\chi_{\text {eff. }}[$ [77] Their experimental data were collected by SAXS on PEO- $b$-PS diblock copolymers blended with $\mathrm{LiCF}_{3} \mathrm{SO}_{3}, \mathrm{LiClO}_{4}$ and $\mathrm{LiAsF}_{6}$ salts. Considering the strong segregation condition, i.e. $\chi N>100$, the theory predicts that:

$$
d^{*} \sim \chi_{e f f}^{1 / 6}
$$

Note that $\chi$ for the neat copolymer was obtained from the literature. In this approximation, the volume occupied by the salt is accounted but changes in statistical segment length upon salt addition are not. Also, the neat sample is in the intermediate regime $(\chi N \sim 18$ at room temperature) where $d^{*}$ is expected to vary from $\chi^{1 / 5}$ to $\chi^{1 / 6}$, inducing a slight overestimation of $\chi_{\text {eff. }}$. Nonetheless, a linear dependence of $\chi_{\text {eff }}$ versus salt concentration is found, which is in agreement with the observations of Balsara and coworkers while exploiting the shift in the phase boundaries.[78] The experimental data of Balsara and coworkers, were obtained by SAXS on PEO- $b$-PS diblock copolymers blended with LiTFSI salt. In that case, the Leibler's theory is used to predict the $\chi$ at the ODT using the following expression:

$$
\left(\chi_{e f f} N\right)_{O D T}=S
$$

where $N=\varphi_{P S} N_{P S}+\varphi_{P E O} N_{P E O}$, with $\varphi_{x}$ and $N_{x}$ respectively the volume fraction and the number of monomers of the specified phase $x . S$ is a constant that depends on $\varphi_{E O}(e . g . S=$ 10.5 for $\varphi_{E O}=0.5$ ). It is important to note that this approximation ignores the temperature dependence of $\chi$. In a second paper the same authors improved their approximation taking into account for composition fluctuations.[79]

In summary, the studies dedicated to the salt concentration effect on $\chi_{\text {eff }}$ reveal systematically a linear relationship which can be expressed as:[80]

$$
\chi_{e f f}=\chi+m \cdot r
$$


where $\chi$ is the intrinsic Flory-Huggins parameter for the salt-free BCP, $r$ is the molar ratio between ions and the solvating monomers. The slope $m$ depends on the anion and block chemical natures. For a PS- $b$-PEO diblock copolymer blended with $\mathrm{LiAsF}_{6}, \mathrm{LiClO}_{4}$ and $\mathrm{LiCF}_{3} \mathrm{SO}_{3} m$ are 5.70, 5.53 and 3.90 respectively. [77] For PS-b-PMMA diblock copolymers blended with $\mathrm{LiCl}, m$ is about $10^{-4}$, [76] and for PS- $b$-PEO diblock copolymers blended with LiTFSI $m$ is 1.56 . [78, 79]. Moreover, $m$ can be estimated considering the dielectric constants of the two phases, the size of the ions ( $m$ decreases with increasing anion radius) and the statistical segment lengths of the polymer chains.[81]

\section{Conclusion}

This paper gives an overview on the essential inputs of scattering techniques, with an emphasis on small angle neutron scattering, on the structural investigations of block copolymers based electrolytes. Two kinds of system were discussed: (i) block copolymers ionomer membranes used in fuel cell and (ii) block copolymers solid polyelectrolytes used in secondary batteries. The literature on neutron scattering is relatively rich concerning the ionomer membranes, which can be explained by the possibility to perform easily contrast variation with various $\mathrm{H}_{2} \mathrm{O}-\mathrm{D}_{2} \mathrm{O}$ mixtures. On the other hand, SANS was rarely exploited to probe the morphology of solid polyelectrolytes used in batteries, SAXS is generally preferred. That opens up a vast field of SANS investigations using, for instance, precise polymer deuteration to probe the morphologies, the salt location, the dead zones, etc. in classical polymer / salt blend electrolytes; but also on more recent and promising systems, such as single ion polymer electrolytes.

\section{Annex}

A. Small angle scattering spectra collected on the BCP samples reveal structural peaks, whose $q$-positions are the signatures of the morphologies. Here, we give the expected $q$ positions for three current morphologies: (i) the $1 \mathrm{D}$ lamellar structure, (ii) the $2 \mathrm{D}$ hexagonal packing of cylinders, and (iii) $3 \mathrm{D}$ cubic arrangement of spheres. The relative peak position is defined as $q_{\text {peak }} / q^{*}$ with $q_{\text {peak }}$ the $q$-position of a specific higher order Bragg diffraction peak and $q^{*}$ the $q$-position of the first order Bragg diffraction peak.

For the lamellar structure the peaks position are given by:[12]

$$
q_{h}=2 \pi h / a
$$

with $h$ the first Miller indice and $a$ the lattice dimension. Then the relative peak positions are expected to follow the sequence: $1,2,3,4,5$, etc. and the lamella-to-lamella distance is expressed as:

$$
a=2 \pi / q^{*}
$$

For the hexagonal packing of cylinders the peak positions are given by the expression:[12]

$$
q_{h k}=4 \pi \sqrt{h^{2}+h k+k^{2}} / \sqrt{3} a
$$


with $h$ and $k$ the first and second Miller indices and $a$ the lattice dimension. The relative peak positions are expected to follow the sequence: $1, \sqrt{ } 3, \sqrt{4}, \sqrt{7}, \sqrt{9}$, etc. and the cylinderto-cylinder distance is expressed as:

$$
a=4 \pi / \sqrt{3} q^{*}
$$

For the cubic arrangement of spheres, the peak positions are given by the expression:[12]

$$
q_{h k l}=2 \pi \sqrt{h^{2}+k^{2}+l^{2}} / a
$$

with $h, k$ and $l$, the Miller indices and $a$, the lattice dimension. The relative peak positions are expected to follow the sequence: $1, \sqrt{2}, \sqrt{3}, \sqrt{ } 4, \sqrt{5}$, etc. and the sphere-to-sphere distance is given by:

$$
a=2 \pi / q^{*}
$$

B. The Kinning and Thomas model[54] is expressed as a product of the form factor for polydisperse spheres, $P$, and the Percus-Yevick structure function, $S_{P Y}$, describing their short-range order in a matrix:[82]

$$
I_{K T}(q)=\Delta^{2} \cdot N \cdot P(q ; \bar{R}, \sigma) \cdot S_{P Y}\left(q ; R_{H S}, \phi_{H S}\right)
$$

where $\Delta^{2}$ is the square of the contrast (i.e. SLDs difference between the spheres and the matrix) and $N$ the number of the scattering particles. It is important to note that in the present scattering intensity formalism, the contrast parameter, $\Delta^{2}$ is not contained in the form factor unlike presented in expression (2). This form factor simplification is allowed since homogeneous spheres are considered. $\overline{\mathrm{R}}$ is the mean sphere radius and $\sigma$ is the rootmean-square deviation from the mean size. $R_{H S}$ and $\phi_{H S}$ are respectively the hard sphere radius and the hard spheres volume fraction. $2 R_{H S}$ corresponds to the closest approach between two spheres.

$P$ is expressed as

$$
P(q ; \bar{R}, \sigma)=\frac{\int_{0}^{\infty} p(R ; \bar{R}, \sigma) f^{2}(q, R) d R}{\int_{0}^{\infty} p(R ; \bar{R}, \sigma) d R}
$$

with $f(\mathrm{q}, \mathrm{R})$ the sphere form factor of radius $\mathrm{R}$, given by

$$
f(q ; R)=\left(\frac{4 \pi}{3}\right) R^{3} \frac{3}{(q R)^{3}}[\sin (q R)-q R \cos (q R)]
$$

and $p(R)$ is a probability function considering a Schultz distribution,

$$
p(R ; \bar{R}, Z)=\left(\frac{Z+1}{\bar{R}}\right)^{Z+1} \frac{R^{Z} \exp \left[-\frac{Z+1}{\bar{R}} R\right]}{\Gamma(Z+1)}
$$

in which $\Gamma(x)$ is the Gamma function and $\mathrm{Z}+1=\left(\frac{\bar{R}}{\sigma}\right)^{2}$.

Then, 


$$
P(q ; \bar{R}, Z)=8 \pi^{2} \bar{R}^{6}(Z+1)^{-6} Q^{Z+7} G_{1}(Q)
$$

with $Q=\frac{(Z+1)}{q \bar{R}}$ and $G_{l}$ is defined as

$$
\begin{gathered}
G_{1}(Q)=Q^{-(Z+1)}-\left(4+Q^{2}\right)^{-\frac{Z+1}{2}} \cos \left[(Z+1) \tan ^{-1} \frac{2}{Q}\right] \\
-2(Z+1)\left(4+Q^{2}\right)^{-(Z+2) / 2} \sin \left[(Z+2) \tan ^{-1} \frac{2}{Q}\right] \\
+(Z+2)(Z+1)\left(Q^{-(Z+3)}+\left(4+Q^{2}\right)^{-(Z+3) / 2} \cos \left[(Z+3) \tan ^{-1} \frac{2}{Q}\right]\right)
\end{gathered}
$$

The Percus-Yevick structure function, $S_{P Y}$, is given by

$$
S_{P Y}\left(q ; R_{H S}, \phi_{H S}\right)=\frac{1}{1+24 \phi_{H S} G_{2}(A) / A}
$$

in which $A=2 q R_{R H}$ and $G_{2}$ is given by the following expression,

$$
\begin{aligned}
G_{2}(A) & =\frac{\alpha}{A^{2}}(\sin A-A \cos A)+\frac{\beta}{A^{3}}\left(2 A \sin A+\left(2-A^{2}\right) \cos A-2\right) \\
& +\frac{\gamma}{A^{5}}\left(-A^{4} \cos A+4\left[\left(3 A^{2}-6\right) \cos A+\left(A^{3}-6 A\right) \sin A+6\right]\right)
\end{aligned}
$$

where $\alpha, \beta$ and $\gamma$ are

$$
\begin{gathered}
\alpha=\frac{\left(1+2 \phi_{H S}\right)^{2}}{\left(1-\phi_{H S}\right)^{4}} \\
\beta=\frac{-6 \phi_{H S}\left(1+\phi_{H S} / 2\right)^{2}}{\left(1-\phi_{H S}\right)^{4}} \\
\gamma=\frac{\frac{1}{2} \phi_{H S}\left(1+2 \phi_{H S}\right)^{2}}{\left(1-\phi_{H S}\right)^{4}}
\end{gathered}
$$

C. The Teubner-Strey model[55] is used to fit broad peaks which are the signature of irregularly shaped domains with short-range order. This model has two parameters defining two characteristic lengths: $d$ a characteristic separation length of the domains and $\xi$ a correlation length that describes the extent of correlation of the domain arrangement. Then the Teubner-Strey scattered intensity is given by

$$
I_{T S}(q ; d, \xi)=\frac{8 d^{4} \pi}{\xi\left[16 \pi^{4}+8 d^{2} \pi^{2}\left(\xi^{-2}-q^{2}\right)+d^{4}\left(\xi^{-2}+q^{2}\right)\right]}
$$

D. The Flory-Huggins parameter, $\chi$, can be extracted from the scattering data through the random phase approximation theory (RPA), i.e. the correlation hole in the disordered regime.[2] To do so, the correlation hole peak is fitted with a structure factor, $S(q)$, which accounts for the concentration fluctuations:[9]

$$
\frac{1}{S(q)}=\frac{S_{A A}+S_{B B}+2 S_{A B}}{S_{A A} S_{B B}-S_{A A}^{2}}-\chi
$$

where $S_{i j}$ correspond to the Fourier transform of the density-density correlation function for the component $i$ and $j$. The $S_{i j}$ can be expressed in term of Debye functions, when considering large block length, then: 


$$
\begin{gathered}
S_{A A}=N g(f, x) \\
S_{B B}=N g(1-f, x) \\
S_{A B}=\frac{N}{2}(g(1, x)-f(f, x)-g(1-f, x))
\end{gathered}
$$

with $g(f, x)=\frac{2}{x^{2}}\left(f x+e^{-f x}-1\right), x$ is given by $x=q^{2} R_{g}^{2}$, where $R_{g}$ denotes the whole copolymer radius of gyration (i.e. $R_{g}=N b^{2} / 6$, if a unique statistical segment length $b$ is considered). The number fraction of A segments $f$, is expressed $f=N_{A} /\left(N_{A}+N_{B}\right)$. The general trend is that the $\chi$ value dictates the peak intensity maximum and also the peak width. On the other hand, the statistical segment length $b$ determines the $q$-position of the peak.

\section{Acknowledgements}

I am grateful to M. Wolf, F. Cousin, M. Månssson and I. Grillo for their kind invitation to the French \& Swedish Winter School in Neutron Scattering (Uppsala, 2016). Also, I would like to thanks my supervisors and colleagues O. Diat, G. Gebel, B. Frisken, S. Holdcroft, R. Mezzenga, J. Ruokolainen, D. Mecerreyes, T. Bowden and D. Brandell for our fruitful collaborations. Finally, I thank LLB, ILL, SINQ, NIST-CNR and ISIS for the neutrons beamtime allocations.

\section{References}

1. F. S. Bates, G. H. Fredrickson, Physics Today 52, 32 (1999)

2. L. Leibler, Macromolecules 13, 1602 (1980)

3. F. S. Bates, G. H. Fredrickson, Annual Review of Physical Chemistry 41, 525 (1990)

4. M. W. Matsen, F. S. Bates, Macromolecules 29, 1091 (1996)

5. M. W. Matsen, Physical Review Letters 99, 148304 (2007)

6. M. W. Matsen, The European Physical Journal E 36, 44 (2013)

7. C. J. Clarke, A. Eisenberg, J. La Scala, M. H. Rafailovich, J. Sokolov, Z. Li, S. Qu, D. Nguyen, S. A. Schwarz, Y. Strzhemechny, B. B. Sauer, Macromolecules 30, 4184 (1997)

8. A. V. G. Ruzette, P. Banerjee, A. M. Mayes, M. Pollard, T. P. Russell, R. Jerome, T. Slawecki, R. Hjelm, P. Thiyagarajan, Macromolecules 31, 8509 (1998)

9. B. Stuehn, A. R. Rennie, Macromolecules 22, 2460 (1989)

10. Y. Mai, A. Eisenberg, Chem Soc Rev 41, 5969 (2012)

11. R. Borsali, R. Pecora, Soft-Matter Charactezation (Springer, 2008)

12. S. Förster, A. Timmann, M. Konrad, C. Schellbach, A. Meyer, S. S. Funari, P. Mulvaney, R. Knott, The Journal of Physical Chemistry B 109, 1347 (2005)

13. Y. Yin, Q. Du, Y. Qin, Y. Zhou, K.-i. Okamoto, Journal of Membrane Science 367, $211(2011)$

14. D. X. Luu, E.-B. Cho, O. H. Han, D. Kim, The Journal of Physical Chemistry B 113, $10072(2009)$

15. X. Zhang, L. Sheng, T. Hayakawa, M. Ueda, T. Higashihara, Journal of Materials Chemistry A 1, 11389 (2013)

16. E. A. Weiber, S. Takamuku, P. Jannasch, Macromolecules 46, 3476 (2013)

17. F. Ng, D. J. Jones, J. Rozière, B. Bauer, M. Schuster, M. Jeske, Journal of Membrane Science 362, 184 (2010)

18. G. Gebel, O. Diat, Fuel Cells 5, 261 (2005) 
19. S. Lyonnard, École thématique de la Société Française de la Neutronique 11, 177 (2010)

20. L. Rubatat, $\mathrm{PhD}$ thesis, New structural model for Nafion membranes, the reference polymer for low temperature fuel cells (UJF, Grenoble, 2003).

21. P. Debye, J. H.R. Anderson, H. Brumberger, J. Appl. Phys. 28, 679 (1957)

22. H. Brumberger, J. Goodisman, J. Appl. Crist. 16, 83 (1983)

23. L. Rubatat, G. Gebel, O. Diat, Macromolecules 37, 7772 (2004)

24. T. D. Gierke, G. E. Munn, F. C. Wilson, J. of Poly. Sc. 19, 1687 (1981)

25. T. D. Gierke, W. Y. Hsu, Am. Chem. Soc., 283 (1982)

26. W. Y. Hsu, T. D. Gierke, Journal of Membrane Science 13, 307 (1983)

27. L. Rubatat, A. L. Rollet, G. Gebel, O. Diat, Macromolecules 35, 4050 (2002)

28. K. Schmidt-Rohr, Q. Chen, Nature Materials 7, 75 (2008)

29. L. Rubatat, O. Diat, Macromolecules 40, 9455 (2007)

30. K.-D. Kreuer, G. Portale, Advanced Functional Materials 23, 5390 (2013)

31. J. Baruchel, J. L. Hodeau, M. S. Lehmann, J. R. Regnard, C. Schlenker, Neutron and synchrotron radiation for condensed matter studies (Springler-Verlag, 1993)

32. R. A. Weiss, A. Sen, C. L. Willis, L. A. Pottick, Polymer 32, 1867 (1991)

33. R. A. Weiss, A. Sen, L. A. Pottick, C. L. Willis, Polymer 32, 2785 (1991)

34. X. Lu, W. P. Steckle, R. A. Weiss, Macromolecules 26, 5876 (1993)

35. X. Y. Lu, W. P. Steckle, B. Hsiao, R. A. Weiss, Macromolecules 28, 2831 (1995)

36. K. A. Mauritz, R. F. Storey, D. A. Reuschle, N. B. Tan, Polymer 43, 5949 (2002)

37. K. A. Mauritz, R. F. Storey, D. A. Mountz, D. A. Reuschle, Polymer 43, 4315 (2002)

38. Y. A. Elabd, C. W. Walker, F. L. Beyer, J. Membr. Sci. 231, 181 (2004)

39. Y. A. Elabd, E. Napadensky, J. M. Sloan, D. M. Crawford, C. W. Walker, J. Membr. Sci. 217, 227 (2003)

40. A. Mokrini, C. D. Rio, J. L. Acosta, Solid State Ionics 166, 375 (2004)

41. A. Mokrini, J. L. Acosta, Polymer 42, 9 (2001)

42. S. Mani, R. A. Weiss, C. E. Williams, S. F. Hahn, Macromolecules 32, 3663 (1999)

43. D. Gromadzki, P. Cernoch, M. Janata, V. Kudela, F. Nallet, O. Diat, P. Stepanek, European Polymer Journal 42, 2486 (2006)

44. M. J. Park, K. H. Downing, A. Jackson, E. D. Gomez, A. M. Minor, D. Cookson, A. Z. Weber, N. P. Balsara, Nano Letters 7, 3547 (2007)

45. L. Rubatat, Z. Q. Shi, O. Diat, S. Holdcroft, B. J. Frisken, Macromolecules 39, 720 (2006)

46. Z. Shi, S. Holdcroft, Macromolecules 38, 4193 (2005)

47. Z. Q. Shi, Phd, Synthesis and Characterization of Proton Conducting, FluorineContaining Block Copolymers (Simon Fraser University, Burnaby, 2004).

48. Z. Shi, S. Holdcroft, Macromolecules 37, 2084 (2004)

49. J. B. Hayter, J. Penfold, J. Phys. Chem. 88, 4589 (1984)

50. G. Gebel, Elaboration et etude structurale de membranes ionomeres perfluorees a partir de solutions (Universite Joseph Fourier, Grenoble, 1989).

51. A. L. Rollet, O. Diat, G. Gebel, The J. of Phys. Chem. 106, 3033 (2002)

52. A. C. C. Yang, R. Narimani, Z. Zhang, B. J. Frisken, S. Holdcroft, Chemistry of Materials 25, 1935 (2013)

53. R. Narimani, A. C. C. Yang, E. M. W. Tsang, L. Rubatat, S. Holdcroft, B. J. Frisken, Macromolecules 46, 9676 (2013)

54. D. J. Kinning, E. L. Thomas, Macromolecules 17, 1712 (1984)

55. M. Teubner, R. Strey, The Journal of Chemical Physics 87, 3195 (1987) 
56. L. Rubatat, C. Li, H. Dietsch, A. Nykänen, J. Ruokolainen, R. Mezzenga, Macromolecules 41, 8130 (2008)

57. C. Monroe, J. Newman, Journal of The Electrochemical Society 152, A396 (2005)

58. R. Bouchet, S. Maria, R. Meziane, A. Aboulaich, L. Lienafa, J.-P. Bonnet, T. N. T. Phan, D. Bertin, D. Gigmes, D. Devaux, R. Denoyel, M. Armand, Nature Materials 12, 452 (2013)

59. R. Meziane, J. P. Bonnet, M. Courty, K. Djellab, M. Armand, Electrochimica Acta 57, 14 (2011)

60. R. Bouchet, S. Maria, R. Meziane, A. Aboulaich, L. Lienafa, J.-P. Bonnet, T. N.

T. Phan, D. Bertin, D. Gigmes, D. Devaux, R. Denoyel, M. Armand, Nature Materials 12, 452 (2013)

61. J. Mindemark, L. Imholt, D. Brandell, Electrochimica Acta 175, 247 (2015)

62. Y. Wang, F. Fan, A. L. Agapov, T. Saito, J. Yang, X. Yu, K. Hong, J. Mays, A. P. Sokolov, Polymer 55, 4067 (2014)

63. X. Yu, J. B. Bates, G. E. Jellison, F. X. Hart, Journal of The Electrochemical Society 144, 524 (1997)

64. J. Y. Song, Y. Y. Wang, C. C. Wan, Journal of The Electrochemical Society 147, $3219(2000)$

65. P. E. Stallworth, J. J. Fontanella, M. C. Wintersgill, C. D. Scheidler, J. J. Immel, S. G. Greenbaum, A. S. Gozdz, Journal of Power Sources 81-82, 739 (1999)

66. S. Cheng, D. M. Smith, C. Y. Li, Macromolecules 47, 3978 (2014)

67. C. D. Robitaille, D. Fauteux, Journal of The Electrochemical Society 133, 315 (1986)

68. Z. Gadjourova, Y. G. Andreev, D. P. Tunstall, P. G. Bruce, Nature 412, 520 (2001)

69. S. K. Fullerton-Shirey, J. K. Maranas, Macromolecules 42, 2142 (2009)

70. H.-G. Peng, M. Tyagi, K. A. Page, C. L. Soles, in Polymers for Energy Storage and Delivery: Polyelectrolytes for Batteries and Fuel Cells. (American Chemical Society, 2012), vol. 1096, chap. 5, pp. 67.

71. D. Devaux, D. Glé, T. N. T. Phan, D. Gigmes, E. Giroud, M. Deschamps, R. Denoyel, R. Bouchet, Chemistry of Materials 27, 4682 (2015)

72. I. Nakamura, Z.-G. Wang, Soft Matter 8, 9356 (2012)

73. R. Bouchet, T. N. T. Phan, E. Beaudoin, D. Devaux, P. Davidson, D. Bertin, R. Denoyel, Macromolecules 47, 2659 (2014)

74. A.-V. r. G. Ruzette, P. P. Soo, D. R. Sadoway, A. M. Mayes, Journal of The Electrochemical Society 148, A537 (2001)

75. A. Bergfelt, L. Rubatat, R. Mogensen, D. Brandell, T. Bowden, Polymer 131, 234 (2017)

76. J.-Y. Wang, W. Chen, T. P. Russell, Macromolecules 41, 4904 (2008)

77. W.-S. Young, T. H. Epps, Macromolecules 42, 2672 (2009)

78. N. S. Wanakule, A. Panday, S. A. Mullin, E. Gann, A. Hexemer, N. P. Balsara, Macromolecules 42, 5642 (2009)

79. N. S. Wanakule, J. M. Virgili, A. A. Teran, Z.-G. Wang, N. P. Balsara, Macromolecules 43, 8282 (2010)

80. F. Xue, S. Jiang, RSC Advances 3, 23895 (2013)

81. I. Nakamura, N. P. Balsara, Z. G. Wang, Phys Rev Lett 107, 198301 (2011)

82. J. K. Percus, G. J. Yevick, Physical Review 110, 1 (1958) 